AIAA-98-4763

\title{
Progress Toward Using Sensitivity Derivatives in a High-Fidelity Aeroelastic Analysis of a Supersonic Transport
}

\author{
and \\ Jaroslaw Sobieszczanski-Sobieski ${ }^{\dagger}$ \\ NASA Langley Research Center, Hampton, Virginia 23681
}

\begin{abstract}
Several government/commercial off-the-shelf modular software packages have been combined to perform

plinary optimization (MDO). Results obtained for the aeroelastic analysis and aerodynamic optimization cases are consistent with expectations and serve as a benchmark for future results that will incorporate sensitivity derivatives from the computational
\end{abstract}

\section{Introduction}

Multidisciplinary design optimization (MDO) methods developed in the past decade have not been id $\mathrm{l} d \mathrm{td} \mathrm{b}$ th $\mathrm{US}$ i d t i

plications typically involve tens to hundreds of variables and hundreds of constraints. In addition, a single high-fidelity aerodynamic or structural analysis of an aircraft configuration requires several CPU hours on a supercomputer Thus traditional optimization
The goal of this research project is to address the issue of computational expense in MDO through the use of computational fluid dynamics (CFD) solvers and finite element (FE) analysis/optimization soft

FE solvers have recently become available due to the implementation of three techniques. These are (1) the development of stand-alone sensitivity equation solvers for computational aerodynamics such as SENSE (see Godfrey and Cliff [1]) (2) the use of au-

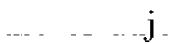
(... R R....... [- [- [Haftka and Gurdal [5]). Both of these methods yield sensitivity derivative data at a lower computational expense than would be incurred if using traditional finite difference-based techniques to estimate sensi- an optimal aircraft design. In such a scenario the computational expense of applying MDO to realistic design problems quickly becomes prohibitive.
In progress toward the goal of high-fidelity MDO for aircraft configurations, an initial capability has been developed to perform multidisciplinary analysis for static aeroelastic problems along with singledisciplinary aerodynamic optimization In this re

\footnotetext{
*Postdoctoral Fellow, National Research Council. Member AIAA

$\dagger$ Leader, Computational Aerosciences Team, and Coordinator of Multidisciplinary Design Optimization Activities, Research and Technology Group. Fellow AIAA

Copyright (c) 1998 by Anthony A. Giunta. Published by the American Institute of Aeronautics and Astronautics, Inc., with permission.
} 
between the two disciplines is accomplished through a stand-alone software package which integrates the aerodynamic pressures to form aerodynamic loads, and then transfers the loads to the FE model via a suite of interpolation schemes. While such an approach is certainly not new (cf., Tzong et al [7], and Chen et al [8]), the uniqueness of this research stems from (1) the use of government/commercial off-theshelf software for the CFD solver, FE solver, grid generator, and loads transfer, and (2) the goal of using available sensitivity derivatives to reduce the computational expense of high-fidelity aeroelastic analysis and optimization problems.

This paper contains the background information on the suite of software developed for this project along with initial results for aeroelastic analysis and aerodynamic optimization cases performed without incorporating sensitivity derivatives. These data form the benchmark results to which future analysis and optimization cases, performed with sensitivity derivatives, will be compared. The methods incorporating sensitivity derivatives in multidisciplinary analysis and multidisciplinary optimization are described in Sections 2.2 and 2.3.

The remainder of this paper is arranged in the following manner. In Section 2 the aeroelastic analysis problem is introduced. Section 3 contains a descrip-

\section{$\begin{array}{llll}\mathrm{y} & \mathrm{y} & \mathrm{p} & \mathrm{p}\end{array}$}

presented in Section 5. A summary of this work along with items for future work are contained in Sections 6 and 7 , respectively.

\section{Aeroelastic Analysis Sensitivity Derivatives}

with

\subsection{Aeroelastic Problem Formulation}

In static aeroelasticity the aircraft designer seeks to predict the deflections which an elastic structure will undergo when subject to time-invariant aerodynamic loads. The aerodynamic component of this aerostructural interaction is modeled as a solution of the governing fluid flow equations, $\mathbf{R}$, in the form

$$
\mathbf{R}\left[\mathbf{Q}\left(\mathbf{X}_{\mathbf{a}}\right), \mathbf{X}_{\mathbf{a}}(\mathbf{D})\right]=\mathbf{0}
$$

where $\mathbf{D}$ is a set of design parameters describing the aircraft geometry, $\mathbf{X}_{\mathbf{a}}$ is the discretized representation of the flow field surrounding the aircraft model, and $\mathbf{Q}$ is the solution of the state (flow field) variables on $\mathbf{X}_{\mathbf{a}}$. The vector of aerodynamic forces on the surface of the aircraft, $\mathbf{F}_{\mathbf{a}}$, is represented as

$$
\mathbf{F}_{\mathbf{a}}=\mathbf{F}_{\mathbf{a}}\left[\mathbf{Q}\left(\mathbf{X}_{\mathbf{a}}\right), \mathbf{X}_{\mathbf{a}}(\mathbf{D})\right]
$$

The structural component of the aero-structural interaction is modeled as

$$
\mathbf{F}_{\mathbf{s}}=\mathbf{F}_{\mathrm{s}}\left[\mathbf{F}_{\mathbf{a}}, \mathbf{X}_{\mathbf{s}}(\mathbf{D})\right]
$$

and

$$
\mathbf{F}_{\mathrm{s}}=\mathbf{K u} \text {. }
$$

In Equation $3, \mathbf{F}_{\mathrm{s}}$ is the vector of applied forces (including structural weight) on the discretized structural model, $\mathbf{X}_{\mathbf{s}}$. In Equation $4, \mathbf{K}$ is the stiffness matrix of the discretized undeformed structure, and $\mathbf{u}$ is the unknown vector of nodal displacements due to the applied forces.

In general, the discretization of the solid boundary of the aerodynamic grid does not coincide with the discretization of the outer surface of the structural grid. Thus, a mechanism is needed to transfer the aerodynamic loads from $\mathbf{X}_{\mathbf{a}}$ to $\mathbf{X}_{\mathbf{s}}$. In addition, another mechanism is needed to map the structural deflections, u, to the design parameters, D. Once these are available Equations 1-4 may be solved using an iterative scheme.

Note that while nonlinear aerodynamic loads are used in this study, the structural model is evaluated

during the iterative process of solving Equations 1 - 4. Consequently, during each iteration the vector of structural loads, $\mathbf{F}_{\mathbf{s}}$, is applied to the undeformed structural model. The static wing deflections for a HSCT at cruise conditions should exhibit linear behavior (i.e., small wing tip deflections with respect to the wingspan). This assumption of linearity may not be valid for off-cruise conditions which are not considered in this research.

\subsection{Sensitivity Derivatives in Analysis}

Much of the computational expense in repeatedly solving Equations 1 - 4 occurs in using a CFD solver to satisfy Equation 1 as $\mathbf{D}$ varies. It is here that the sensitivity derivatives are useful in reducing the computational expense of high-fidelity aeroelastic analyses. This is accomplished by approximating $\mathbf{Q}\left[\mathbf{X}_{\mathbf{a}}(\mathbf{D}+\Delta \mathbf{D})\right]$ rather than solving Equation 1 for $\mathbf{D}+\Delta \mathbf{D}$. This approximation, denoted by $\tilde{\mathbf{Q}}$, is performed using a first-order Taylor series expansion around $\mathbf{D}$ of the form

$$
\tilde{\mathbf{Q}}\left[\mathbf{X}_{\mathbf{a}}(\mathbf{D}+\Delta \mathbf{D}] \approx \mathbf{Q}\left[\mathbf{X}_{\mathbf{a}}(\mathbf{D})\right]+\frac{\partial \mathbf{Q}}{\partial \mathbf{D}} \Delta \mathbf{D}\right.
$$


where via the chain rule

$$
\frac{\partial \mathbf{Q}}{\partial \mathbf{D}}=\frac{\partial \mathbf{Q}}{\partial \mathbf{X}_{\mathbf{a}}} \frac{\partial \mathbf{X}_{\mathbf{a}}}{\partial \mathbf{D}} .
$$

The sensitivity derivative matrix $\partial \mathbf{Q} / \partial \mathbf{D}$ may be extracted from the output of CFD solver such as CFL3D.ADII $[2,9]$, where CFL3D.ADII is a version of CFL3D [10] to which the automatic differentiation tool ADIFOR has been applied. In CFL3D.ADII the sensitivity derivative matrix is obtained at the same time the governing fluid flow equations are solved. Alternatively, the sensitivity derivative matrix may be obtained using a post-CFD sensitivity equations solver such as SENSE [1]. In SENSE, the user provides the state variables obtained from a CFD solution along with a perturbed aerodynamic grid, and SENSE returns the sensitivity derivative matrix.

The approximation of the state variables, $\tilde{\mathbf{Q}}$, is then updated as $\mathbf{D}$ changes for each iteration of the aeroelastic analysis process. Eventually the Taylor series approximation of the state variables will degrade and one must solve Equation 1 to obtain accurate state variables. Thus, a sequence of Taylor series approximations of $\mathbf{Q}$ is performed until convergence is attained.

Such sequential approximation methods have been used by numerous researchers in the past including McQuade et al [11], and Hutchison et al [12]. However, these and similar approaches have used finite difference techniques to estimate the sensitivity derivatives, rather than analytically obtained sensitivity derivatives.

\subsection{Sensitivity Derivatives in MDO}

Available sensitivity derivatives also permit the use of high-fidelity analysis tools in multidisciplinary optimization, in a manner similar to their use in highfidelity analysis described above. One such use of the sensitivity derivatives is in the formulation of the Global Sensitivity Equations (GSE) developed by Sobieski [13]. In a coupled aero-structural problem, the aerodynamic derivatives in the GSE matrices would be obtained using either CFL3D.ADII or SENSE, and the structural derivatives would be obtained from a FE solver such as MSC/NASTRAN [14] or GENESIS [15], both of which calculate sensitivity deriva- nite difference methods to estimate the terms in the GSEs.

Another MDO formulation that would benefit from the use of sensitivity derivatives is the collaborative optimization (CO) strategy of Kroo et al [18]. The CO method employs a bilevel optimization technique in which a system-level optimizer ensures compatibility of the competing disciplines (e.g., aerodynamics, structures, and performance) by prescribing target values that each discipline-level optimizer must meet. Thus, the discipline-level optimizers must be computationally efficient since they are implemented numerous times during a $\mathrm{CO}$ execution.

For both the GSE-based and the CO strategies described above, the computational expense of using finite difference methods and high-fidelity CFD and FE solvers is prohibitive. The availability of sensitivity derivatives has the potential to make the use of high-fidelity solvers in the GSE-based and CO approaches computationally affordable.

\section{Analysis Models}

\subsection{Baseline HSCT Model}

The HSCT model used in this research is a generic wing/fuselage configuration extensively studied by numerous researchers including Barthelemy et al [16], Dovi et al [17], Hutchison et al [12], and Giunta et al [19]. The mission performance, and hence the economic viability, of such an aircraft hinges on the trade-offs among the often competing engineering disciplines involved in aircraft design. Thus, the HSCT is particularly well-suited to a multidisciplinary design approach. In this research, the inter-disciplinary couplings are limited to those of aerodynamics and structural mechanics.

The original vehicle used in this study was sized to accommodate 252 passengers for a range of 5,500 nautical miles, and a cruise Mach number of 2.4. [16]. Here, the baseline HSCT model is simplified to a wing/fuselage configuration. That is, the horizontal tail, vertical tail, and engine nacelles are removed. While simplified, this model contains sufficient complexity to be of interest for aeroelastic analysis and aerodynamic optimization. Details on the aerodynamic and structural models of this HSCT are pro- then be used for optimization studies such as those performed by Barthelemy et al [16] and Dovi et al [17]. Note that both of these studies employed fi-
The baseline HSCT configuration is parameterized using a technique originally developed by Hutchison et al [12] to describe generic aircraft wing and fuselage 
shapes. For this research project, modifications to Hutchison's technique have been made to accommodate variable camber and twist along the span. In this study the area-ruled fuselage and the wing/fuselage fairing are held fixed and 64 parameters are used to model the variations in planform and wing shape.

the wing break ( $\left.Y_{b r e a k}\right)$, and the y-location of the wing tip $\left(Y_{\text {tip }}\right)$. Completing the planform specification is the root chord with a fixed length of 144.87 $f t$.

parameter $\left(R_{p}\right)$, and the chordwise location of maximum thickness for the entire wing $\left(C_{\max }\right.$ thk. $)$. In addition, the $\mathrm{t} / \mathrm{c}$ ratio of the wing root is fixed at 2.96 percent. In this parameterization the $\mathrm{t} / \mathrm{c}$ ratio $\mathrm{f}$ th $\mathrm{i} \quad \mathrm{i} \quad \mathrm{li} \quad \mathrm{l}$ b t th $\mathrm{t} \quad \mathrm{h} \quad \mathrm{d}$

the radius-to-chord ratio, $r / t$, given by

$$
r / t=1.1019\left[\left(R_{p} / 6\right)(t / c)\right]^{2}
$$

where $R_{p}$ is the non-dimensional leading edge radius parameter.

The camber and twist are specified at each of the 13 airfoils using a cubic polynomial of the form

$$
z_{k}=c_{1, k}(x / c)+c_{2, k}(x / c)^{2}+c_{3, k}(x / c)^{3},
$$

where $(x / c)$ is the normalized chordwise distance specified at 20 locations along each chord, and $k=$ $1, \ldots, 13$. Here the 39 polynomial coefficients control the spanwise camber and twist distribution

\section{Analysis and Optimization Software}

\subsection{Software Overview}

The software suite developed in this research in- sign Optimization) [20] developed at NASA Langley, (2) the Euler/Navier-Stokes solver CFL3D [10] also developed at NASA Langley, (3) the finite element analysis and optimization package GENESIS [15] de-

$$
\text { l d b VMA E i i I d (4) h }
$$

stitute and funded by the U.S. Air Force.

This collection of G/COTS software is loosely coupled using UNIX shell scripts and some in-house software created to parse input and output files as

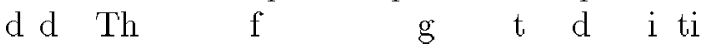

Aeroelastic analysis is performed by coupling the software modules in an arrangement depicted by Figure 2 where the file interface routines have been omitted for clarity. The arrows in Figure 2 denote the passing of data among the analysis routines.

In Figure 2 the box labeled "Geometry Manipulator" contains the in-house developed software which translates the parametric representation of the HSCT model into file formats for the G/COTS modules. For input into CSCMDO an aerodynamic surface grid is generated in the HESS format, and for input into GENESIS a finite element model is created in the MSC/NASTRAN Bulk Data format, with which GENESIS is compatible. Ideally, this geometry translation would be performed with G/COTS software such as Pro-Engineer, AutoCAD, or CATIA. However, a number of constraints made it impractical to

and Sun workstations, However, all CEL $3 \mathrm{D}$ GENESIS analyses are performed exclusively on SGI workstations to permit accurate comparisons of expended computational resources. Timings reported 
all calculations performed thus far have been done serially. Future efforts will incorporate parallel com-

Prior to performing an aerodynamic analysis, a volume grid is generated based on the aerodynamic surface grid using the G/COTS module CSCMDO. This software tool is an automated, multi-block, structured three-dimensional volume grid generator developed by members of the Geometry Laboratory (GEOLAB) at the NASA Langley Research Center. In addition to grid generation, CSCMDO also has the capability to modify an existing volume grid based on perturbations to the underlying surface grid. It is in this capacity that CSCMDO is used here, where a surface grid and a volume grid for the baseline HSCT were provided to the authors by GEOLAB. The user then provides a perturbed aerodynamic surface grid to CSCMDO which redistributes the grid points throughout the computational volume using a transfinite interpolation scheme. The volume grid generated by CSCMDO is written to a file in PLOT3D format, which is a common format used by numerous CFD solvers. This efficient grid regeneration capability facilitates the use of CSCMDO in aeroelastic analysis and aerodynamic optimization where the volume grid must be updated repeatedly as the structure deforms or is modified during the optimization process.

The CFD solver used in this study is the G/COTS program known as CFL3D. This software was developed at the NASA Langley Research Center and currently is maintained by members of the Aerodynamic and Acoustic Methods Branch. CFL3D is a time-dependent, Reynolds-averaged, thin-layer Navier-Stokes flow solver for use with two- or threedimensional structured grids. A third-order accurate finite-volume spatial discretization is employed in CFL3D, along with both mesh sequencing and multigrid techniques for convergence acceleration. In addition, multiblock connection strategies are possi-

tions are solved on a volume grid with a $\mathrm{C}-\mathrm{O}$ discretization having the dimensions $121 \times 41 \times 61$, in the streamwise, circumferential, and surface normal directions, respectively. The volume grid is divided is a total of approximately 300,000 grid points in the model (see Figure 3).

conditions correspond to those obtained from the U.S. Standard Atmosphere tables listed by Shevell [24] for an end-of-cruise altitude of $63,000 \mathrm{ft}$. Here, freestream density, pressure and temperature are $0.000198 \mathrm{lb} \cdot \mathrm{sec}^{2} / \mathrm{ft}^{4}, 130.86 \mathrm{lb} / \mathrm{ft}^{2}$, and $392^{\circ} \mathrm{R}$, respectively. The reference area used in calculating $C_{L}$ and $C_{D}$ is based on the planform area of the HSCT configuration. For the baseline HSCT halfspan model the reference area is $4609.65 \mathrm{ft}^{2}$. In this model the coordinate axes are aligned such that the $\mathrm{x}$-axis points in the positive streamwise direction (i.e., from nose-to-tail) and the $y$-axis points in the spanwise direction from the fuselage centerline to the wing tip.

For all of the calculations presented here the angle-of-attack is held constant at $3.5^{\circ}$ with zero sideslip. Thus, symmetry in the $x-z$ plane permits the use of a half-span model, with appropriate boundary conditions along the plane of symmetry. The flow tangency condition is enforced along the solid surfaces of the wing/fuselage model and freestream conditions are imposed on the outer boundary of the computational grid.

During the initial solution of the Euler equations for the aerodynamic model, the entire flow field is initialized to freestream conditions. Both mesh sequencing and two-level V-cycle multigrid are employed to accelerate convergence. The initial flow field solution is obtained after 400 iterations which is sufficient to converge the residual error parameter in CFL3D by five orders of magnitude. The typical computational cost for this initial solution is approximately 1.3 CPU hours.

Subsequent solutions of the Euler equations are obtained using the restart capability in CFL3D. Here, the flow field values are initialized to converged flow field values of a previous CFL3D solution instead of

The convergence history and analysis results of CFL3D are output in a variety of files and formats. One output option is to generate files in the PLOT3D format which contain the grid point locations on the 


\subsection{Structural Model and Analysis}

The FE solver used in this study is GENESIS which performs structural analysis and optimization, where both element sizing and shape optimization are possible. The model definition format in GENESIS is identical to that of MSC/NASTRAN, which is somewhat of a de-facto standard in structural analysis. The similarity between GENESIS and MSC/NASTRAN is useful since many software packages are written to read FE models in NASTRAN format

Balabanov et al [26]). A copy of this structural model generator was provided to the authors and was modified for use in this research. The wing/fuselage model of the HSCT is comprised of a fixed number and arrangement of spar and rib elements. The layout of the structural elements is based on the planform and airfoil thickness parameters used to represent the HSCT configuration.

The finite element model of the HSCT configurations has 206 nodes and 1130 elements with a total of 1254 degrees-of-freedom. Note that due to structural symmetry only the starboard portion of the model is constructed. The FE model contains triangular membrane elements for the fuselage and wing skins, along with rod elements for the spar cap and rib caps. The spar and rib webs are modeled with a combination of shear panels and rod elements. The material for all structural elements is titanium alloy Ti- $6 \mathrm{Al}-4 \mathrm{~V}$. The structural layout of the baseline HSCT wing/fuselage model is shown in Figure 4.

Some sources of non-structural wing weight are incorporated into the half-span FE model. These sources include $44,580 \mathrm{lb}$ for engine weight, $7,800 \mathrm{lb}$ for the main landing gear weight, and $19,800 \mathrm{lb}$ of reserve fuel stored in the wings. These data were obtained using the FLOPS (Flight Optimization System) [27] program to analyze the baseline HSCT configuration considered here. Note that the engine weight was concentrated at four FE nodes on the
Mises yield stress criteria and local buckling constraints under load conditions approximating a $2.5 \mathrm{~g}$ pull-up maneuver at Mach 2.4. In this element sizing there are 40 variables where 26 of these are skin panel thickness parameters (i.e., 13 panels on each of the upper and lower body surfaces), 12 variables define spar cap areas, and two variables describe the rib cap areas. Typically, this sizing optimization requires 15 20 CPU minutes on a Silicon Graphics workstation.

After the initial sizing in GENESIS the element di $\quad \mathrm{i} \quad \mathrm{h}$ ld fi $\mathrm{d} i$ th $b \quad \mathrm{t}$

ESIS structural analysis requires approximately 50 CPU seconds.

\subsection{Aerodynamic Loads Transfer}

The software used to calculate the aerodynamic loads and to transfer them to the structural model is known as the Fluids and Structures Interface Toolkit (FASIT) developed by Smith et al [21, 22]. Currently, FASIT is maintained by the Air Force Research Laboratory at the Wright-Patterson Air Force Base.

FASIT is ideally suited for the loosely coupled approach to aeroelasticity, as it accepts several widelyused file formats employed by CFD and FE solvers. In this research, the PLOT3D format is used to input the grid and flow field data from CFL3D and the NASTRAN Bulk Data format is used to input the FE surface grid. FASIT provides a suite of interpolation schemes for transferring the aerodynamic loads from the CFD surface grid to the FE surface grid. The thin plate spline method of Duchon [28] was used in this study, as recommended in the FASIT User's Manual [22]. Note that the interpolation methods in FASIT conserve the total force and moments for all three axes when transferring loads from the CFD grid to the FE grid.

The loads transferred from FASIT to the FE surface grid are written in the NASTRAN Bulk Data format. Since this NASTRAN format is compatible

surfaces. However, no attempt was made to remove structural elements to model the landing gear bay. The fuel weight was equally distributed to $118 \mathrm{FE}$ nodes in the inboard wing section and in the leading edge strake.

Prior to an aeroelastic analysis the spar, rib, and skin elements are sized using GENESIS to meet von

\subsection{Structural Deformation}

From GENESIS an output file is obtained which contains the nodal displacements of the FE surface grid. A simple Fortran program was written to transfer the nodal displacements to the original (undeformed) FE 
surface grid to obtain the grid coordinates of the deformed structure. A Mathematica [29] program was written to calculate the change in camber/twist variables (see Equation 8) and to extract the $\mathrm{z}$-ordinates of the leading edge at each of the 13 airfoil sections which define the wing. The output from the Mathematica routine is a file containing the parameters which are needed by the geometry manipulation software to begin the next iteration in the aeroelastic analysis.

\subsection{Aeroelastic Analysis}

Due to the nonlinearity of the aero/structural interaction the aeroelastic analysis involves an iterative scheme whereby the aerodynamic and structural analyses are performed repeatedly until both the aerodynamic loads and the structural deflections reach convergence (see Figure 2). Following the methods of Chipman et al [30] and Tzong et al [7], a constant factor under-relaxation method is used to accelerate convergence of this iterative process. A value of 0.7 for the relaxation parameter is used for all aeroelastic calculations described here. The computational cost of the aerodynamic analyses dominates the total CPU time involved in obtaining a converged aeroelastic analysis.

\subsection{Aerodynamic Optimization}

The modularity of the CSCMDO and CFL3D codes permits a simple reorganization of the software for use in aerodynamic optimization. Here, the commercial optimization software package DOT (Design Optimization Tools) [31] is coupled to the aerodynamic analysis software using an interface program as shown in Figure 5. The sequential quadratic programming (SQP) method in DOT is used for the aerodynamic optimization case presented here.

\section{Results}

\section{$5.1 \quad$ Aeroelastic Analysis}

The static aeroelastic analysis case is performed to calculate the external shape of the deformed HSCT structure under $1.0 \mathrm{~g}$ Mach 2.4 cruise conditions. Recall that a constant factor under-relaxation method, with a value of 0.7 , is used to accelerate convergence of this iterative process. Typically, convergence is obtained in six iterations (2.7 CPU hours) when under-relaxation is used as compared to 19 iterations (6.1 CPU hours) without the relaxation method as is shown in Figure 6. The case with relaxation was terminated when the wing tip deflection was less than $0.05 \mathrm{ft}$, while the case without relaxation was terminated after 19 iterations due to lack of convergence.

Results for the aeroelastic analysis with relaxation are shown in Figures 7 and 8. In Figure 7 the final deformed wing is shown in comparison to the solid outline of the undeformed wing. Note that the zaxis in this figure has been scaled to exaggerate the wing deflection for easier viewing. Figure 8 shows the aeroelastic deformation of the HSCT wing at the wing tip, wing break ( $y=33.7 \mathrm{ft})$, and wing root. At the $1.0 \mathrm{~g}$ cruise conditions considered here the zdirection wing tip deflection of approximately $1.5 \mathrm{ft}$ is reasonable. For this HSCT wing/fuselage model at a fixed angle-of-attack of $3.5^{\circ}$ (relative to the fuselage centerline), this static aeroelastic analysis reveals a reduction in lift of about 5.7 percent compared to the lift produced by the rigid wing/fuselage model.

\subsection{Aerodynamic Optimization}

The aerodynamic optimization problem utilizes a tenvariable subset of the 64 parameters which describe the wing planform and shape. In particular, the six planform variables and the four thickness variables described in Section 3.2 are used in this sample problem. Note that the camber parameters are held fixed at their nominal values throughout the optimization process. The initial, minimum, and maximum values for the 10 variables are listed in Table 1.

The optimization problem is formulated to minimize the drag coefficient subject to two constraints: (1) lift $\geq 210,000 \mathrm{lb}$, and, (2) a geometric constraint on the chord length at the wing leading edge break location. The geometric constraint prevents the optimizer from creating a planform shape where the inboard trailing edge angle is positive and the outboard trailing edge angle is zero or negative. Such a trailing edge is undesirable from a structural standpoint because of the complexity of the load path (i.e., spar layout) that it creates.

The results of the optimization are listed in $\mathrm{Ta}-$ ble 2 and demonstrate a reduction of three drag counts $\left(\Delta C_{D}=0.0003\right)$ between the initial and optimal HSCT configurations. This corresponds to a decrease of approximately 5.8 percent of the total inviscid drag.

The computational expense of this optimization is about $16 \mathrm{CPU}$ hours on a single-processor SGI R10000 workstation where virtually all of the expense is in the CFD analyses. The convergence history for both $C_{D}$ and the $C_{L}$ constraint is shown in Figure 9 .

A planform view of the initial and optimal wing 
planforms is shown in Figure 10, and the wing tip, wing break, and wing root airfoils are shown in Figure 11. Recall that camber variables are not included in this optimization problem. Therefore, all of the wing shape differences are due to differences in the 10 planform and thickness variables.

\section{Conclusions}

The goal of this work is the development of a highfidelity MDO capability for aircraft configurations. Progress toward this goal is shown here through the use of modular G/COTS software for static aeroelastic analysis and aerodynamic optimization cases

derivatives, obtained from the CFD and FE solvers in the software suite, into multidisciplinary analysis and optimization cases.

\section{Future Work}

A number of items remain for current and future work on this project. The first of these is the use of sensitivity derivatives in a sequential approximation strategy (described in Section 2.2) for the aeroelastic analysis and optimization cases. Subsequently, the sensitivity derivatives will be incorporated into the GSE-based and CO frameworks for MDO as described in Section 2.3. In conjunction with these activities, parallel computation will be used to reduce the wall-clock computational expense of these MDO cases through the use of the parallel computing capabilities in CFL3D and GENESIS.

\section{Acknowledgements}

The authors wish to thank members of the Multidisciplinary Design Optimization Branch, the Aerodynamic and Acoustic Methods Branch, and the Geometry Laboratory (GEOLAB) at NASA Langley Research Center for their assistance with this project. In addition, the authors are grateful for the assistance of Lt. Joel Luker, USAF, who provided the FASIT software package.

This work was performed while the first author held a National Research Council Research Associateship at NASA Langley. Funding for this research is provided by the National Research Council and by the High Performance Computing and Communications Program in Computational Aerosciences.

\section{References}

[1] Godfrey, A. G. and Cliff, E. M. "Direct Calculation of Aerodynamic Force Derivatives: A Sensitivity-Equation Approach," AIAA Paper 98-0393 (Jan. 1998).

[2] Taylor, A. C., III, Oloso, A., and Newman , J. C., III "CFL3D.ADII (Version 2.0): An Efficient, Accurate, General-Purpose Code for Flow Shape-Sensitivity Analysis," AIAA Paper 972204 (1997).

[3] Bischof, C. H., Jones, W. T., Mauer, A., and Samareh-Abolhassani J "Application of Auto-

tion, volume FED 232, pp. 17 22, San Francisco, CA (1995).

[4] Reuther, J., Alonso, J. J., Rimlinger, M. J., and Jameson, A. "Aerodynamic Shape Optimization of Supersonic Aircraft Configurations via an Adjoint Formulation on Distributed Memory Computers," AIAA Paper 96-4045 (Sept. 1996).

[5] Haftka, R. T. and Gurdal, Z. Elements of Structural Optimization, pp. 211-219,305-316, Kluwer, Boston, MA, 3rd edition (1993).

[6] Borland, C. J. "A Multidisciplinary Approach to Aeroelastic Analysis," Comp. Sys. Eng., 1(2-4), $197-209$ (1990).

[7] Tzong, G., Chen, H. H., Chang, K. C., Wu, T., and Cebeci, T. "A General Method for Calculating Aero-Structure Interaction on Aircraft Configurations," in Proceedings of the 6th AIAA/USAF/NASA/ISSMO Symposium on Multidisciplinary Analysis and Optimization, pp. 14-24, Bellevue, WA, AIAA Paper 96-3982 (Sept. 1996).

[8] Chen, H. H., Chang, K. C., Tzong, T., and Cebeci, T. "Aeroelastic Analysis of Wing and Wing/fuselage Configurations," AIAA Paper 98-0907 (Jan. 1998).

[9] Sherman, L., Taylor, A., Green, L., Newman, P., Hou, G., and Korivi, V. "First- and SecondOrder Aerodynamic Sensitivity Derivatives via Automatic Differentiation with Incremental Iterative Methods," J. Comp. Physics, 129, 307-331 (1996). 
namic and Acoustic Methods Branch, NASA Langley Research Center, Hampton, VA, 1st edition (1996).

[11] McQuade, P. D., Eperhardt, S., and Livne, E. "CFD-Based Aerodynamic Approximation Concepts Optimization of a Two-Dimensional Scramjet Vehicle," J. Aircraft, 32(2), 262-269 (1995).

[12] Hutchison, M. G., Unger, E. R., Mason, W. H., and Grossman, B. "Variable-Complexity Aerodynamic Optimization of an HSCT Wing Using Structural Wing-Weight Equations," J. Aircraft, $31(1), 110-116$ (1994).

[13] Sobieszczanski-Sobieski, J. "Sensitivity of Complex, Internally Coupled Systems," AIAA J., 28(1), 153-160 (1990).

[14] Anonymous. MSC/NASTRAN Version 70 Release Guide, Los Angeles, CA (1997).

[15] Vanderplaats, Miura and Associates, Inc., Colorado Springs, CO. GENESIS User's Manual, Version 4.0 (1997).

[16] Barthelemy, J.-F. M., Wrenn, G. A., Dovi, A. R., Coen, P. G., and Hall, L. E. "Supersonic Transport Wing Minimum Weight Design Integrating Aerodynamics and Structures," J. Aircraft, $31(2), 330-338$ (1994).

[17] Dovi, A. R., Wrenn, G. A., Barthelemy, J.F. M., Coen, P. G., and Hall, L. E. "Multidisciplinary Design Integration Methodology for a Supersonic Transport Aircraft," J. Aircraft, $32(2), 290-296$ (1995).

[18] Kroo, I., Altus, S., Braun, R., Gage, P., and Sobieski, I. "Multidisciplinary Optimization Methods for Aircraft Preliminary Design," in Proceedings of the 5th AIAA/USAF/NASA/ISSMO Symposium on Multidisciplinary Analysis and Optimization, pp. 697-707, Panama City Beach, FL, AIAA Paper 94-4325 (Sept. 1994).

[19] Giunta, A. A., Balabanov, V., Haim, D., Grossman, B., Mason, W. H., Watson, L. T., and Haftka, R. T. "Multidisciplinary Optimisation of a Supersonic Transport Using Design of Experiments Theory and Response Surface Modelling," Aeronautical J., 101(1008), 347-356 (1997). timization," in Proceedings of the 12th AIAA Computational Fluid Dynamics Conference, pp. 657-669, San Diego, CA, AIAA Paper 95-1689 (June 1995).

[21] Smith, M. J., Hodges, D., and Cesnik, C. An Evaluation of Computational Algorithms to Interface Between CFD and CSD Methodologies, Flight Dynamics Directorate, Wright Laboratory, Wright-Patterson Air Force Base, OH, Report WL-TR-96-3055 (1995).

[22] Smith, M. J., Hodges, D., and Cesnik, C. Fluids and Structures Interface Toolkit (FASIT), Version 1.0, Georgia Tech Research Institute, Atlanta, GA, Report GTRI A-9812-200 (1996).

[23] Walatka, P. P., Clucas, J., McCabe, R. K., Plessel, T., and Potter, R. FAST User's Guide, Version 2.0, NAS Division, NASA Ames Research Center, Mountain View, CA, Report RND-93010 (1993).

[24] Shevell, R. S. Fundamentals of Flight, pp. 424425, Prentice Hall, Englewood Cliffs, NJ (1989).

[25] Balabanov, V. O. Development of Approximations for HSCT Wing Bending Material Weight Using Response Surface Methodology, Ph.D. thesis, Virginia Polytechnic Institute and State University, Blacksburg, VA (1997).

[26] Balabanov, V., Kaufman, M., Knill, D. L., Haim, D., Golovidov, O., Giunta, A. A., Haftka, R. T., Grossman, B., Mason, W. H., and Watson, L. T. "Dependence of Optimal Structural Weight on Aerodynamic Shape for a High Speed Civil Transport," in Proceedings of the 6th AIAA/USAF/NASA/ISSMO Symposium on Multidisciplinary Analysis and Optimization, pp. 599-612, Bellevue, WA, AIAA Paper 96-4046 (Sept. 1996).

[27] McCullers, L. A. "Aircraft Configuration Optimization Including Optimized Flight Profiles," in Proceedings of the Symposium on Recent Experiences in Multidisciplinary Analysis and $O p$ timization,

Sobieski, J., ed., pp. 396-412, Hampton, VA, NASA CP 2327 (1984).

[28] Duchon, J. Splines Minimizing RotationInvariant Semi-Norms in Sobolev Spaces, pp. 85-100, Springer-Verlag, Berlin, eds. W. Schempp and K. Zeller (1977). 
[30] Chipman, R., Waters, C., and MacKenzie, D. "Numerical Computation of Aeroelastically Corrected Transonic Loads," in Proceedings of the 20th AIAA/ASME/ASCE/AHS Structures,
0766 (April 1979)

[31] Vanderplaats Research \& Development, Inc., Colorado Springs, CO. DOT User's Manual, Version $4.20(1995)$. 
Table 1: Initial values for the $10 \mathrm{HSCT}$ wing variables and the allowable range on each variable.

\begin{tabular}{cccc} 
Variable & Initial & Minimum & Maximum \\
\hline \hline$\Lambda_{I}$ & $74.0^{\circ}$ & $73.0^{\circ}$ & $75.0^{\circ}$ \\
$\Lambda_{O}$ & $45.0^{\circ}$ & $40.0^{\circ}$ & $50.0^{\circ}$ \\
$C_{\text {break }}$ & $42.4 \mathrm{ft}$ & $40.0 \mathrm{ft}$ & $45.0 \mathrm{ft}$ \\
$C_{\text {tip }}$ & $9.3 \mathrm{ft}$ & $7.0 \mathrm{ft}$ & $10.0 \mathrm{ft}$ \\
$Y_{\text {break }}$ & $28.6 \mathrm{ft}$ & $25.0 \mathrm{ft}$ & $30.0 \mathrm{ft}$ \\
$Y_{\text {tip }}$ & $67.3 \mathrm{ft}$ & $65.0 \mathrm{ft}$ & $70.0 \mathrm{ft}$ \\
$(t / c)_{\text {break }}$ & $2.15 \%$ & $1.50 \%$ & $3.00 \%$ \\
$(t / c)_{\text {tip }}$ & $2.36 \%$ & $1.50 \%$ & $3.00 \%$ \\
$R_{p}$ & 4.5 & 3.5 & 5.5 \\
$C_{\text {max. thk. }}$ & $45.0 \%$ & $30.0 \%$ & $48.0 \%$
\end{tabular}

Table 2: Initial and optimal values for the $10 \mathrm{HSCT}$ wing variables.

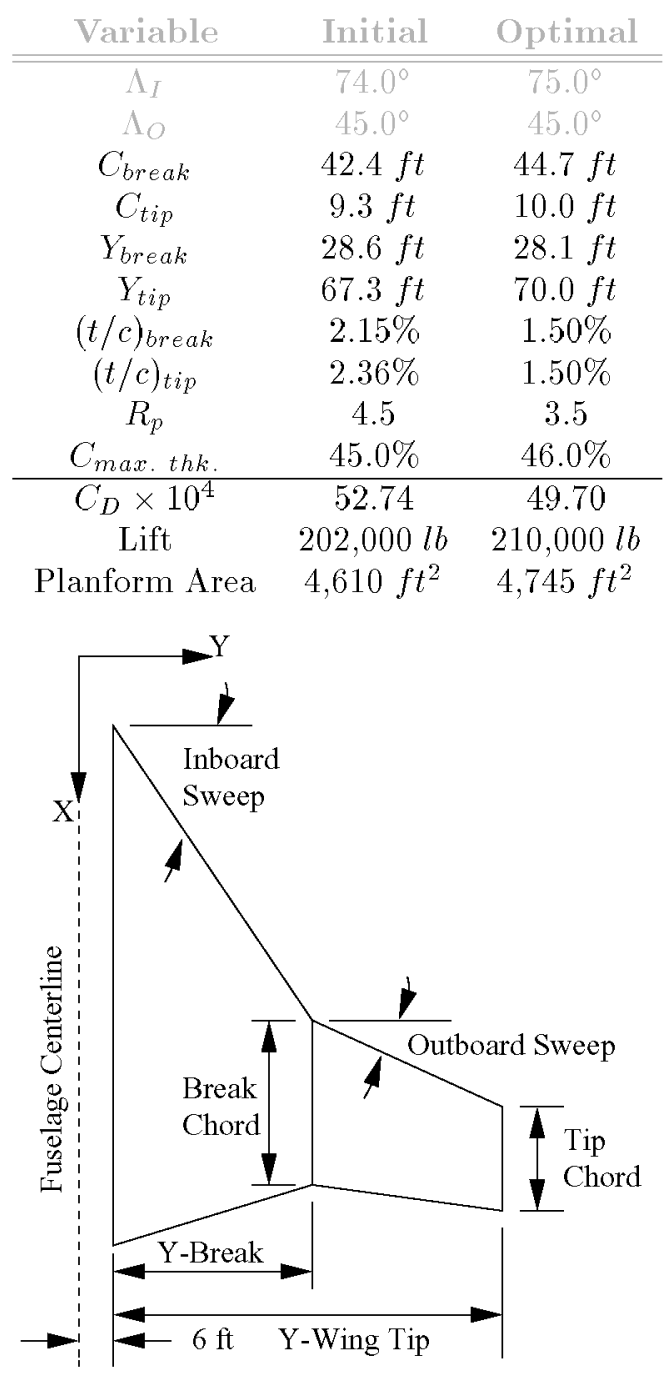

Figure 1: Planform variables for the HSCT wing.

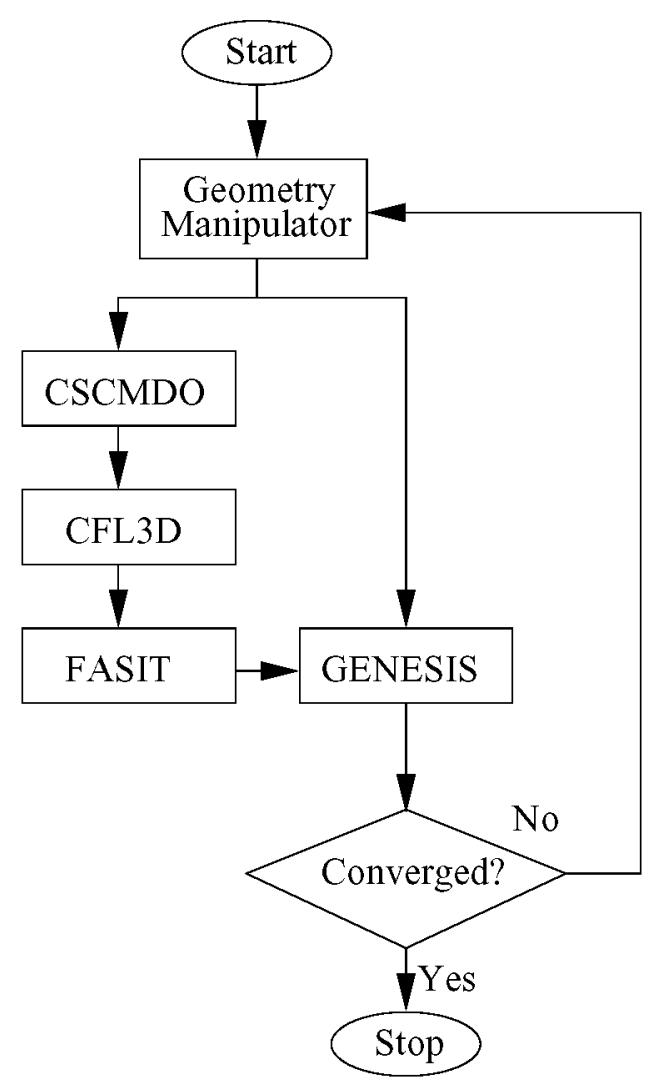

Figure 2: The arrangement of software used to perform static aeroelastic analysis.

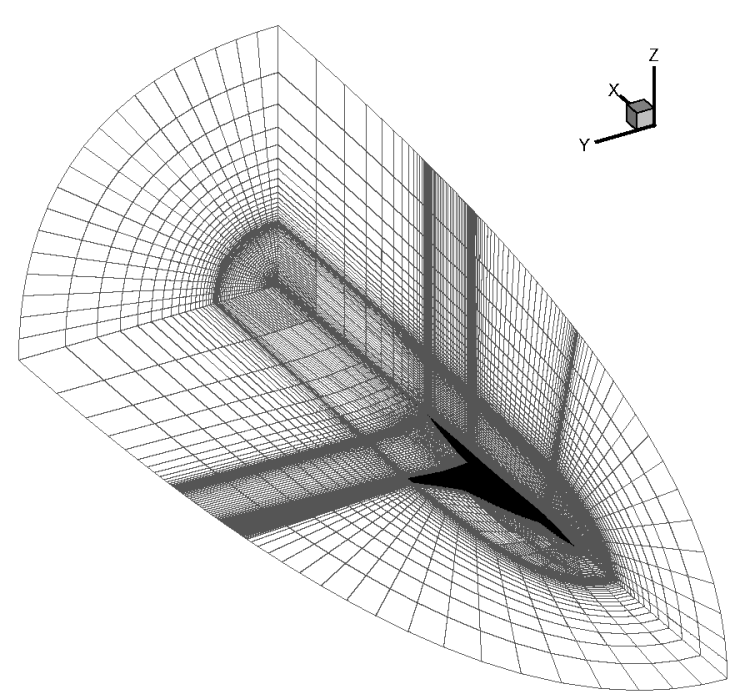

Figure 3: A view of the aerodynamic model of the HSCT showing the surface grid on the starboard wing, the $x-z$ plane of symmetry, and the exit plane. 


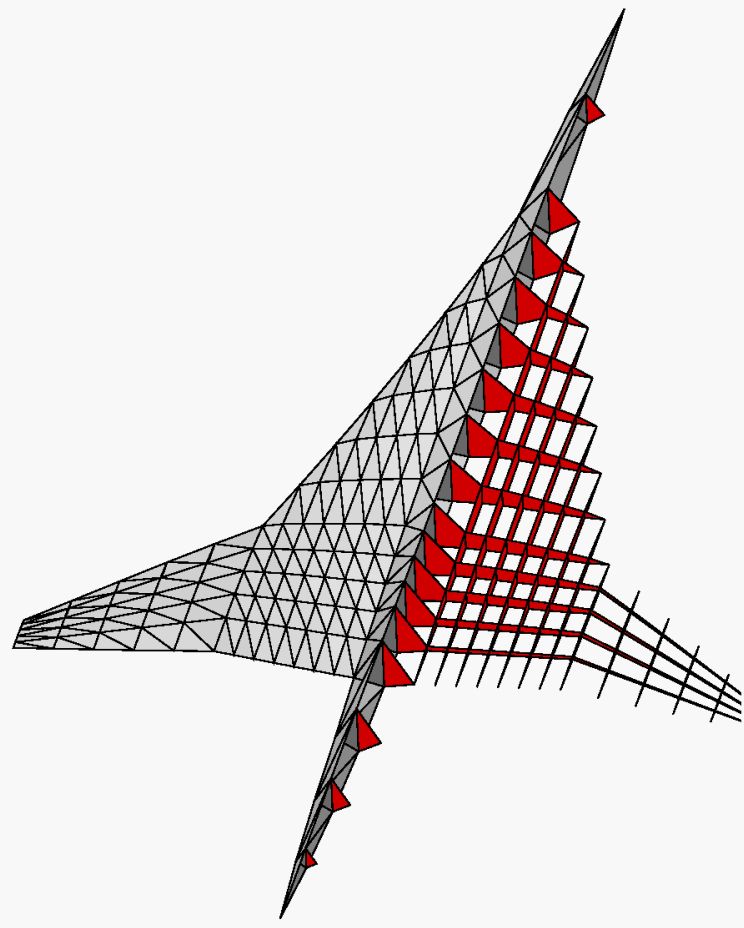

Figure 4: A view of the structural model of the HSCT showing the wing skin elements (port) and the rib/spar elements (starboard).

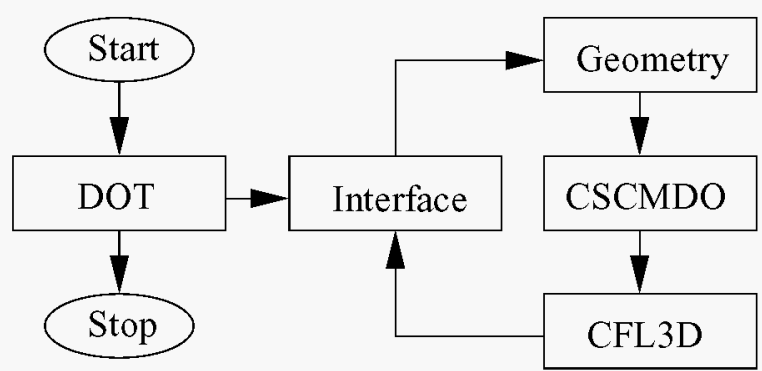

Figure 5: The arrangement of software used to perform aerodynamic optimization.

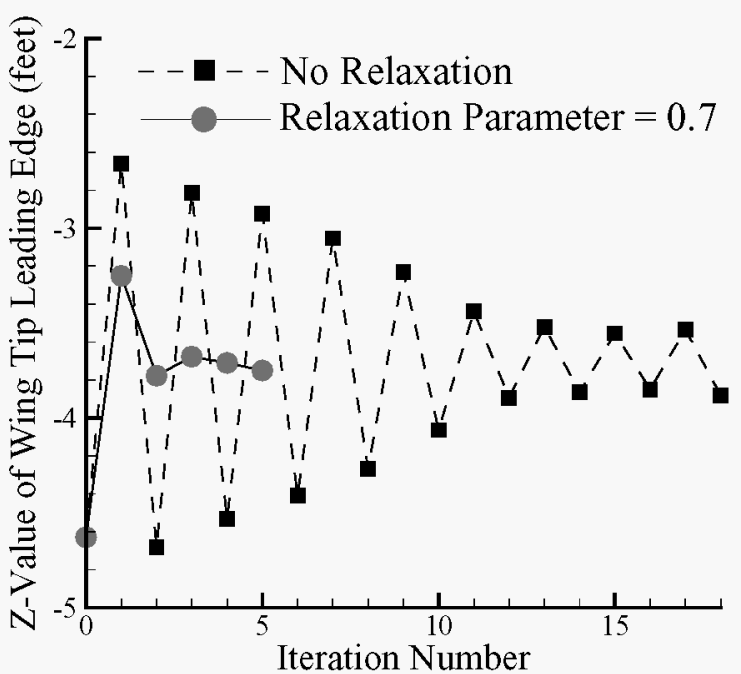

Figure 6: Convergence history of the aeroelastic analysis with and without relaxation.

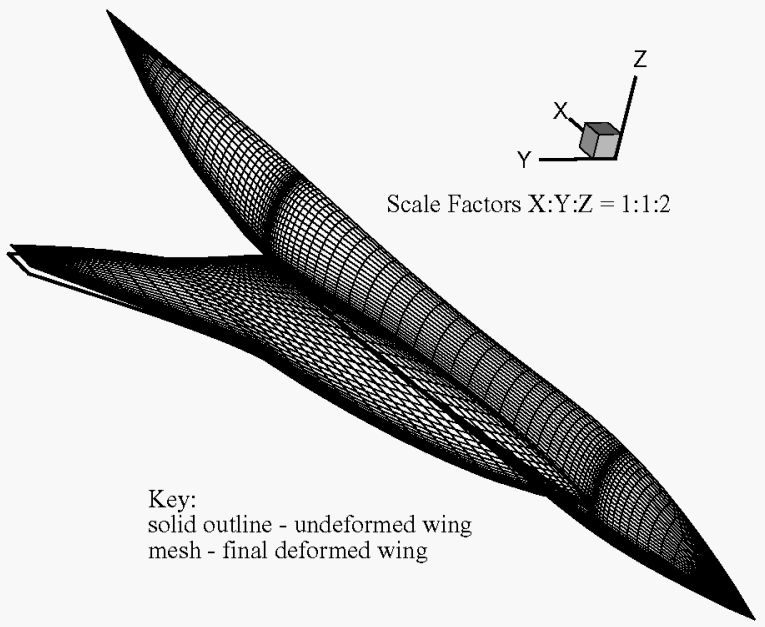

Figure 7: Orthographic view of the deformed wing (mesh) and the undeformed wing (solid outline). Note the $\mathrm{X}: \mathrm{Y}: \mathrm{Z}$ scaling of 1:1:2 used to show the wing deformation. 

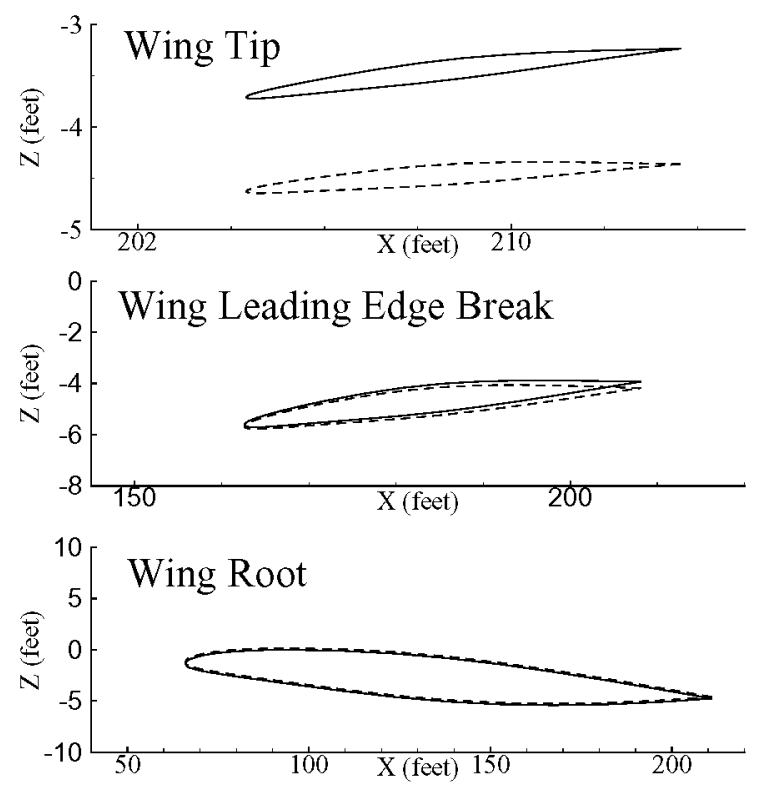

Figure 8: Airfoil sections at the (top-bottom) wing tip, wing break, and wing root for the initial undeformed (dashed) and final deformed (solid) HSCT wing shapes.

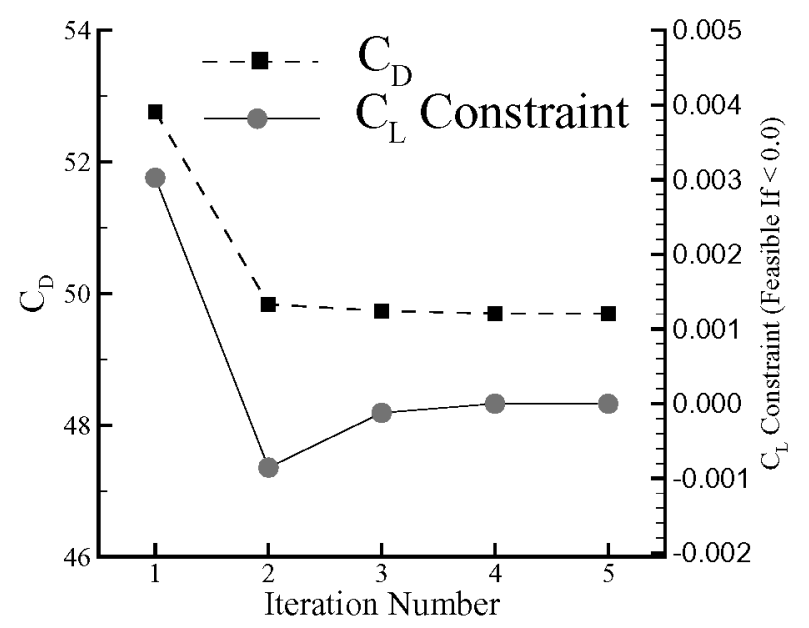

Figure 9: Optimization history for the 10 variable HSCT wing design.

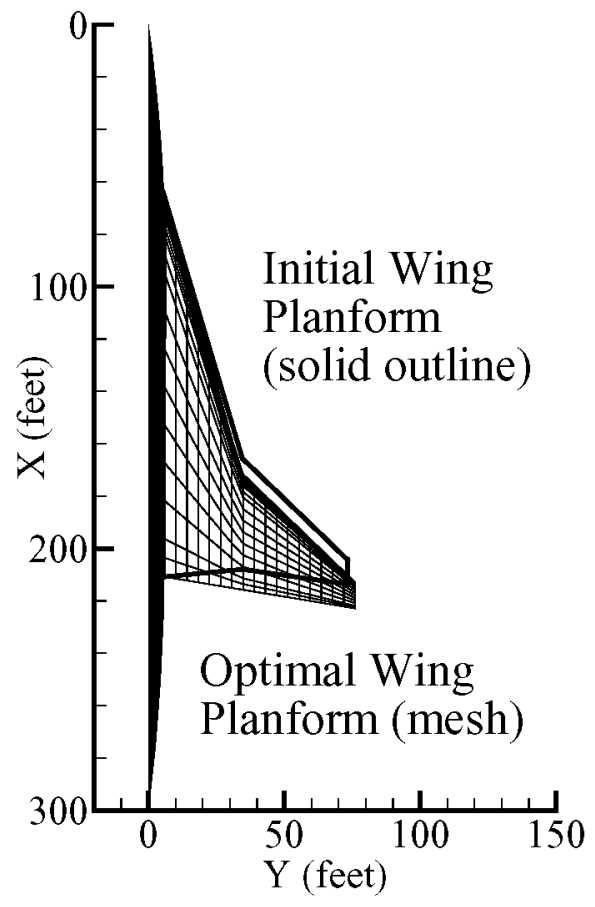

Figure 10: A planform view of the initial and optimal HSCT configurations.
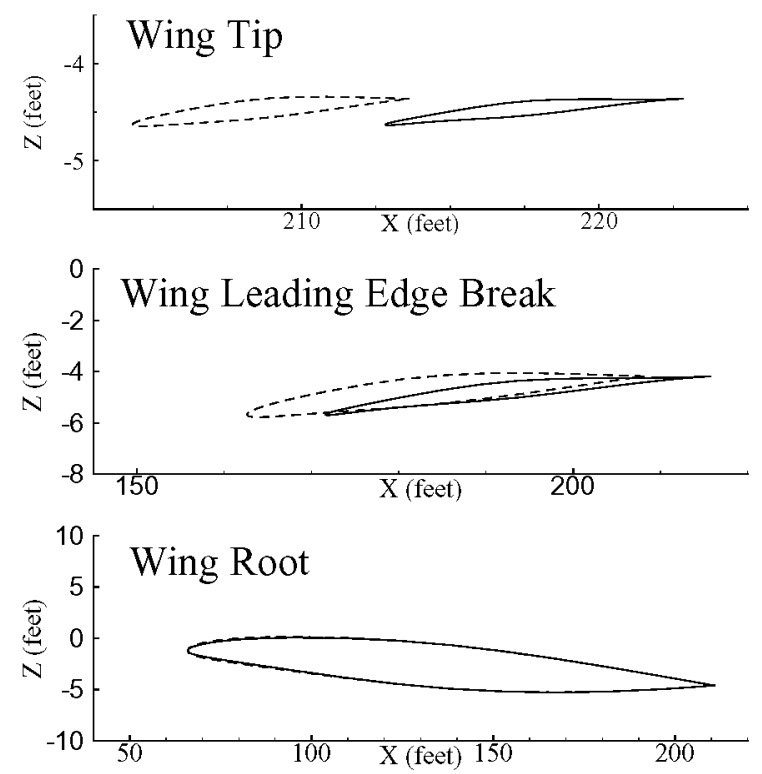

Figure 11: Airfoil sections at the (top-bottom) wing tip, wing break, and wing root for the initial (dashed) and optimal (solid) HSCT wing shapes. 